\title{
Entropy of Classical Histories
}

\author{
Todd A. Brun* \\ Institute for Theoretical Physics, \\ University of California, Santa Barbara, CA 93106-4030 \\ and \\ James B. Hartle \\ Department of Physics, \\ University of California, Santa Barbara, CA 931069530
}

\begin{abstract}
We consider a number of proposals for the entropy of sets of classical coarse-grained histories based on the procedures of Jaynes, and prove a series of inequalities relating these measures. We then examine these as a function of the coarse-graining for various classical systems, and show explicitly that the entropy is minimized by the finest-grained description of a set of histories. We propose an extension of the second law of thermodynamics to the entropy of histories. We briefly discuss the implications for decoherent or consistent history formulations of quantum mechanics.
\end{abstract}

*Current address: Physics Department, Carnegie Mellon University, Pittsburgh, PA 152133890. Email: tbrun@andrew.cmu.edu 
NSF-ITP-97-102

\section{INTRODUCTION}

Entropies are measures of the information missing from a coarse-grained description of a system. Different coarse-grained descriptions give rise to different entropies. If an entropy is low at one time, it will have a general tendency to grow as its coarsegraining is translated forward in time. That is the second law of thermodynamics.

Usually, entropy is constructed from a coarse-grained description at a single moment in time. For example, if all that is known of the state of a system at a particular time is its total energy, the missing information is the entropy of the microcanonical ensemble - a quantity which is independent of time. If all that is known at a time are the expected values of the energy, number, and momentum densities, averaged over volumes large enough to be in local equilibrium, then the missing information per volume is the time-dependent entropy density of hydrodynamics.

The Jaynes procedure [1.2] gives a general method for constructing the entropy of a system at a moment of time. To illustrate, let $M$ be the phase space of a classical system and $\rho(x), x \in M$ be the probability distribution representing the state of the system. Suppose $A(x)$ is a classical quantity whose expected value $\langle A\rangle$ is known, where

$$
\langle A\rangle=\int_{M} d x A(x) \rho(x) .
$$

The missing information $S$ is constructed by maximizing the entropy functional

$$
\mathcal{S}(\tilde{\rho})=-\int_{M} d x \tilde{\rho}(x) \log _{2}[\tilde{\rho}(x)]
$$

over all $\tilde{\rho}(x)$ that imply the same expected value. In symbols,

$$
S=\left.\max _{\tilde{\rho}} \mathcal{S}(\tilde{\rho})\right|_{\langle A\rangle_{\tilde{\rho}}=\langle A\rangle_{\rho}} .
$$


However, entropy need not only apply to coarse-grained alternatives at one moment in time. More generally, one can consider the missing information of a sequence of alternatives at a succession of times. These are the entropies of coarse-grained histories of the system. A variety of such entropies have been described (e.g. [3] [5]) and applied to measures of coarse-graining [4], classicality [6], and effective complexity [7]. In theories which possess a notion of history but lack a fixed notion of time (such as certain formulations of quantum gravity [8]) the missing information of histories may be the only notion of entropy available.

In this paper we examine the entropy of histories for classical stochastic systems - classical systems with a probabilistic law of evolution (including deterministic evolution as a special case). We use Isham's history space [10, [1] and a generalization of the Jaynes procedure to give a unified view of several different kinds of entropies for histories and describe relations among them. We illustrate with numerical calculations in some simple examples. Finally, we describe a modest generalization of the second law of thermodynamics applicable to the entropy of histories and test it in a simple model. Our considerations are almost entirely classical, but in Section V we point the way to generalizations for the quantum mechanical case. 


\section{ENTROPIES OF HISTORIES}

\section{A. Histories and History Space}

We consider classical theories with (most generally) a stochastic evolution law in a space $M$ through a finest-grained net of $N$ times separated by equal intervals $\eta$. For this discussion the space $M$ could be a configuration space of particle positions, a spatial lattice, or a phase space. We denote a point in $M$ by $x$.

The cases when $M$ is a discrete space or a continuous manifold differ only formally, and can to a large extent be treated together by using a common notation. We define

$$
\operatorname{Tr} f(x)=\sum_{x \in M} f(x)
$$

when $M$ is discrete, and

$$
\operatorname{Tr} f(x)=\int_{M} d x f(x)
$$

when $\mathrm{M}$ is continuous. This notation is suggestive for the quantum mechanical case to be treated later. We also define

$$
V \equiv \operatorname{Tr}(I)
$$

where $I$ is the unit function on $M . V$ is an integer when $M$ is discrete and a real number when $M$ is continuous.

A fine-grained history is described by a sequence of $x_{A}, A=1, \cdots, N$ for each of the finest-grained net of times. Histories are therefore naturally thought of as living in a classical "history space" $\mathbf{M}=M \times \cdots \times M$, with one factor for each fine-grained time. A point in $\mathbf{M}$ is denoted by $\mathbf{x}$, and corresponds to a fine-grained history. This is the classical analog of the "history space" introduced by Isham [10, and used so

effectively in quantum theory by Isham and Linden [11,5], and Isham, Linden and Schreckenberg [12]. 
A coarse-grained set of alternative histories is a partition of the set $\mathbf{M}$ of finegrained histories into an exhaustive set of mutually exclusive regions or classes $c_{\alpha}$. Each class is a single coarse-grained history. We can usefully introduce projections onto these regions of $\mathbf{M}$,

$$
\mathbf{P}_{\alpha}(\mathbf{x})= \begin{cases}1 & \mathbf{x} \in c_{\alpha} \\ 0 & \mathbf{x} \notin c_{\alpha}\end{cases}
$$

A sequence of coarse-grained alternatives at a series of times $t_{1}, \cdots, t_{n}$ is an example of a coarse-grained history. Suppose the alternatives at time $t_{k}$ are whether $x$ is in one of a set of regions of $M,\left\{\Delta_{\alpha_{k}}^{k}\right\}, \alpha_{k}=1,2, \ldots$, with volumes $V_{\alpha_{k}}^{k}$. We introduce projections on these regions of $M$,

$$
P_{\alpha_{k}}^{k}(x)= \begin{cases}1 & x \in \Delta_{\alpha_{k}}^{k}, \\ 0 & x \notin \Delta_{\alpha_{k}}^{k}\end{cases}
$$

which satisfy

$$
P_{\alpha_{k}}^{k}(x) P_{\alpha_{k}^{\prime}}^{k}(x)=\delta_{\alpha_{k} \alpha_{k}^{\prime}} P_{\alpha_{k}}^{k}(x)
$$

and

$$
\operatorname{Tr}\left(P_{\alpha_{k}}^{k}\right)=V_{\alpha_{k}}^{k}
$$

In this case, a coarse-grained history is a particular sequence of regions $\alpha \equiv\left(\alpha_{n}, \ldots, \alpha_{1}\right)$ and corresponds to a projection on $\mathbf{M}$ of the form

$$
\mathbf{P}_{\alpha}=I \times \cdots \times P_{\alpha_{n}}^{n} \times I \times \cdots \times P_{\alpha_{1}}^{1} \times \cdots \times I
$$

That is, $\mathbf{P}_{\alpha}$ is the projection on $\mathbf{M}$ with projections $P_{a_{k}}^{k}$ inserted at the times $t_{k}$ and I's at all other times. In the discrete case, the most general projection $\mathbf{P}_{\alpha}$ can always be written as a sum of such chains:

\footnotetext{
${ }^{1}$ The notion of coarse-graining has many specific applications in physics. An anonymous referee suggested [9] as a convenient reference to some of these.
} 


$$
\mathbf{P}_{\alpha}(\mathbf{x})=\sum_{\alpha_{1} \ldots \alpha_{n} \in \alpha} \mathbf{P}_{\alpha_{n} \ldots \alpha_{1}}(\mathbf{x}),
$$

which allows the construction of a narrative for each coarse-grained history. For histories of the form (2.6) it would read: "the system was in $\Delta_{\alpha_{1}}^{1}$ at $t_{1}$, then $\Delta_{\alpha_{2}}^{2}$ at $t_{2}, \ldots$ " In the continuum case there is a corresponding integral.

We assume that there is a probability law for the fine-grained histories, that is, a probability function $\mathbf{W}(\mathbf{x})$ on $\mathbf{M}$. $\mathbf{W}(\mathbf{x})$ satisfies

$$
\mathbf{W}(\mathbf{x}) \geq 0, \quad \text { and } \operatorname{Tr}(\mathbf{W})=1 .
$$

Of course, $\mathbf{W}(\mathbf{x})$ may have special forms in particular circumstances. For example, for a Markov process

$$
\mathbf{W}(\mathbf{x})=p_{\eta}\left(x_{N} \mid x_{N-1}\right) p_{\eta}\left(x_{N-1} \mid x_{N-2}\right) \cdots p_{\eta}\left(x_{2} \mid x_{1}\right) \rho\left(x_{0}\right),
$$

where $\rho\left(x_{0}\right)$ is the distribution at the initial time and $p_{\eta}(x \mid y)$ is the transition probability to arrive at $x$ in a time $\eta$ having started from $y$. If $M$ were a classical phase space, deterministic evolution would be represented by (2.9) with

$$
p_{\eta}(x \mid y)=\delta\left(x-x_{\eta}(y)\right)
$$

where $x_{\eta}(y)$ is the phase-space point $y$ evolved by the time $\eta$.

The probability of a coarse-grained history is

$$
p_{\alpha}=\operatorname{Tr}\left(\mathbf{P}_{\alpha} \mathbf{W}\right) \text {. }
$$

For example, in the case of a sequence of alternatives like (2.3) at a series of times, and a Markovian probability of the form (2.9),

$$
\begin{aligned}
p_{\alpha_{n} \cdots \alpha_{1}}= & \int d x_{n} \cdots \int d x_{1} P_{\alpha_{n}}^{n}\left(x_{n}\right) p\left(x_{n} t_{n} \mid x_{n-1} t_{n-1}\right) P_{\alpha_{n-1}}^{n-1}\left(x_{n-1}\right) \\
& \cdots P_{\alpha_{1}}^{1}\left(x_{1}\right) p\left(x_{1} t_{1} \mid x_{0} t_{0}\right) \rho\left(x_{0}\right) \\
\equiv & \int d x_{0} C_{\alpha_{n} \cdots \alpha_{1}}\left(x_{0}\right) \rho\left(x_{0}\right) .
\end{aligned}
$$

Here $p\left(x^{\prime} t^{\prime} \mid x t\right)$ is the composition of all the $p_{\eta}$ 's from $t$ to $t^{\prime}, t_{0}$ is the initial time, and $C_{\alpha_{n} \cdots \alpha_{1}}\left(x_{0}\right)$ is defined to be the probability of a coarse-grained history $\alpha_{1} \cdots \alpha_{n}$ given that the system is initially at $x_{0}$. 


\section{B. The Entropy of Histories}

The Jaynes construction may now be applied in history space to give an entropy for histories. We introduce the entropy functional'

$$
\mathcal{S}(\mathbf{W})=-\operatorname{Tr}\left(\mathbf{W} \log _{2} \mathbf{W}\right)
$$

(Unable to introduce a bold-faced calligraphic $\mathrm{S}$, we rely on the argument of $\mathcal{S}$ to distinguish this definition from (1.21)).

The history space entropy $S_{h s}\left(\left\{c_{\alpha}\right\}\right)$ of a set of coarse-grained alternative histories is then

$$
\left.S_{h s}\left(\left\{c_{\alpha}\right\}\right) \equiv \max _{\widetilde{\mathbf{W}}} \mathcal{S}(\widetilde{\mathbf{W}})\right|_{\operatorname{Tr}\left(\mathbf{P}_{\alpha} \widetilde{\mathbf{W}}\right)=\operatorname{Tr}\left(\mathbf{P}_{\alpha} \mathbf{W}\right)} .
$$

In words, $S_{h s}$ maximizes the missing information $\mathcal{S}$ over all probability distributions $\widetilde{\mathbf{W}}$ on $\mathbf{M}$ that reproduce the probabilities of the coarse-grained histories $\left\{c_{\alpha}\right\}$ following from $\mathbf{W}$.

The important property of $S_{h s}\left(\left\{c_{\alpha}\right\}\right)$ is that it increases on coarse-graining. Specifically, suppose $\left\{\bar{c}_{\bar{\alpha}}\right\}$ is a coarse-graining of the set $\left\{c_{\alpha}\right\}$. That means that $\left\{\bar{c}_{\bar{\alpha}}\right\}$ is a partition of the $\left\{c_{\alpha}\right\}$ into larger classes, and

$$
\bar{c}_{\bar{\alpha}}=\bigcup_{\alpha \in \bar{\alpha}} c_{\alpha} .
$$

Then, as with any Jaynes type construction,

$$
S_{h s}\left(\left\{c_{\alpha}\right\}\right) \leq S_{h s}\left(\left\{\bar{c}_{\bar{\alpha}}\right\} .\right.
$$

The proof is immediate from (2.14). The constraints for $\left\{c_{\alpha}\right\}$ contain those for $\left\{\bar{c}_{\bar{\alpha}}\right\}$ but there are more of them. The maximum therefore can only be less.

\footnotetext{
${ }^{1}$ In the continuous case, where $\mathbf{W}$ is probability density, rather than a probability, eqn. (2.13) is not generally invariant under dimensional transformations. However, (2.13) is a standard definition. Dimensionally invariant quantities may be obtained by appropriate subtractions, e.g. $-\log _{2} V^{N}$, or better, by rescaling the coordinates so they are dimensionless.
} 
Since the $\mathbf{P}_{\alpha}(\mathbf{x})$ are mutually exclusive projections, an expression for $S_{h s}$ can be derived by carrying out the maximization using Lagrange multipliers to enforce the constraints. The result is

$$
S_{h s}\left(\left\{c_{\alpha}\right\}\right)=-\sum_{\alpha} p_{\alpha} \log _{2} p_{\alpha}+\sum_{\alpha} p_{\alpha} \log _{2} \operatorname{Tr}\left(\mathbf{P}_{\alpha}\right) .
$$

The maximum value of $S_{h s}$ occurs for the coarsest-grained set of histories set where the only history with nonzero probability is $I \times \cdots \times I$. The maximum is

$$
S_{h s}^{\max }=N \log _{2} V .
$$

The minimum (which occurs for completely fine-grained histories) is zero.

Another useful quantity is the Lloyd-Pagels (LP) depth [3], defined as

$$
\begin{aligned}
\mathcal{D}_{L P}\left(\left\{c_{\alpha}\right\}\right) & \equiv S_{h s}^{\max }-S_{h s}\left(\left\{c_{\alpha}\right\}\right) \\
& =\sum_{\alpha} p_{\alpha} \log _{2} p_{\alpha}-\sum_{\alpha} p_{\alpha} \log _{2}\left[\operatorname{Tr}\left(\mathbf{P}_{\alpha}\right) / \operatorname{Tr}(\mathbf{I})\right] .
\end{aligned}
$$

This has a number of useful features. It is a direct measure of the information in a set of histories; it is invariant under dimensional transformations; and it is invariant under refinement of the fine-grained net of times.

To illustrate, consider the entropy of a history consisting of a set of alternatives $\left\{P_{\alpha}\right\}$ at a single moment of time $t$. This is

$$
S_{h s}\left(\left\{P_{\alpha}\right\}\right)=-\sum_{\alpha} p_{\alpha} \log _{2} p_{\alpha}+\sum_{\alpha} p_{\alpha} \log _{2}\left[\operatorname{Tr}\left(P_{\alpha}\right)\right]+(N-1) \log _{2} V .
$$

This is the entropy that would be obtained from the usual Jaynes construction (1.3) with the addition of the constant $(N-1) \log _{2} V$ representing the missing information at all the other moments of time. By contrast, the depth

$$
\mathcal{D}_{L P}\left(\left\{P_{\alpha}\right\}\right)=\sum_{\alpha} p_{\alpha} \log _{2} p_{\alpha}-\sum_{\alpha} p_{\alpha} \log _{2}\left[\operatorname{Tr}\left(\mathbf{P}_{\alpha}\right) / \operatorname{Tr}(\mathbf{I})\right]
$$

is the same as the $-S$ that would be calculated from (1.3), without extra terms. Note that if we use a dimensionally invariant form $S_{h s}$, by subtracting a term $\log _{2} V^{N}$ as suggested above, we would have the simple relationship

$$
S_{h s}\left(\left\{c_{\alpha}\right\}\right)-\log _{2} V^{N}=-\mathcal{D}_{l p}\left(\left\{c_{\alpha}\right\}\right) .
$$




\section{Other Entropies of Histories}

The history space entropy is not the only information measure that can be associated with histories. In the following we discuss some others and the relationships between them.

Isham and Linden's Entropies.

In their seminal paper on entropy in generalized quantum theory [5], Isham and Linden utilize history space to define a one parameter family of entropies based on the decoherence functional $D\left(\alpha, \alpha^{\prime}\right)$ for a decoherent set of coarse-grained histories. Translated into the notation of this paper their definition reads:

$$
I_{x}\left(\left\{c_{\alpha}\right\}\right)=-\sum_{\alpha} p_{\alpha} \log _{2} p_{\alpha}+x \sum_{\alpha} p_{\alpha} \log _{2}\left[\operatorname{Tr}\left(\mathbf{P}_{\alpha}\right) / \operatorname{Tr}(\mathbf{I})\right]
$$

As they show explicitly, for $x \geq 1$ the entropy $I_{x}\left(\left\{c_{\alpha}\right\}\right)$ possesses the important property that it increases under coarse-graining of the decoherent set.

As discussed by Isham and Linden, in the case of non-relativistic quantum mechanics, history space is a repeated tensor product of the Hilbert space of the system - one factor for each time. The $\mathbf{P}_{\alpha}$ are projections on this space and the trace is defined as usual. However, their arguments can be immediately applied to the classical situations we have been discussing. (We shall return to the quantum mechanical case in Section V.) Indeed, any classical problem can be considered as a generalized quantum theory in which all sets of alternative histories decohere automatically: $D\left(\alpha, \alpha^{\prime}\right) \equiv p(\alpha) \delta_{\alpha \alpha^{\prime}}$. The expression (2.23) thus applies immediately in the classical case. The history space entropy $S_{h s}$ we arrived at from the Jaynes construction corresponds to $x=1$, up to a possible overall renormalization. Isham and Linden mainly consider $x=2$, but that should not obscure the fact that our history space entropy, defined through a Jaynes construction, is a special case of those that

they consider. In Section V we will provide a Jaynes construction for this entropy in quantum mechanics. 
Step-by-step entropy.

Consider the special case where the set of coarse-grained histories consists of a sequence of sets of coarse-grained alternatives at a series of times $t_{1}, \cdots, t_{n}$. For generality we assume that these sets are branch dependent, that is, the sets at time $t_{k}$ may depend on the specific choice of sets at previous times $t_{1} \cdots t_{k-1}$. The projections at time $t_{k}$ then have the form

$$
P_{\alpha_{k}}^{k}\left(\alpha_{k-1}, \cdots, \alpha_{1}\right)
$$

At any stage in the sequence $k=1, \cdots, n$, one can construct the Jaynes entropy of the set of alternatives $\left\{P_{\alpha_{k}}^{k}\right\}$ conditional on a particular previous history $\alpha_{k-1}, \cdots, \alpha_{1}$. This is, following (2.20),

$$
\begin{aligned}
S_{k}\left(\left\{P_{\alpha_{k}}^{k}\right\} \mid \alpha_{k-1}, \cdots, \alpha_{1}\right)= & -\sum_{\alpha_{k}} p_{\alpha_{k} \mid \alpha_{k-1}, \cdots, \alpha_{1}} \log _{2} p_{\alpha_{k} \mid \alpha_{k-1}, \cdots, \alpha_{1}} \\
& +\sum_{\alpha_{k}} p_{\alpha_{k} \mid \alpha_{k-1}, \cdots, \alpha_{1}} \log _{2}\left\{\operatorname{Tr}\left[P_{\alpha_{k}}^{k}\left(\alpha_{k-1}, \cdots, \alpha_{1}\right)\right]\right\} .
\end{aligned}
$$

where $p_{\alpha_{k} \mid \alpha_{k-1}, \cdots, \alpha_{1}}$ is the conditional probability for $\alpha_{k}$ given the previous history $\alpha_{k-1}, \cdots, \alpha_{1}$. In terms of joint probabilities this is

$$
p_{\alpha_{k} \mid \alpha_{k-1}, \cdots, \alpha_{1}}=\frac{p_{\alpha_{k}, \cdots, \alpha_{1}}}{p_{\alpha_{k-1}, \cdots, \alpha_{1}}}
$$

Average the conditioned entropies (2.25) over past histories weighted by their probabilities and sum over all the steps from one to $n$ to obtain the step-by-step entropy $S_{s b s}\left(\left\{P_{\alpha_{n}}^{n}\right\}, \cdots,\left\{P_{\alpha_{1}}^{1}\right\}\right)$ :

$$
S_{s b s}\left(\left\{P_{\alpha_{n}}^{n}\right\}, \cdots,\left\{P_{\alpha_{1}}^{1}\right\}\right)=\sum_{k=1}^{n} \sum_{\alpha_{k-1} \cdots \alpha_{1}} p_{\alpha_{k-1} \cdots \alpha_{1}} S_{k}\left(\left\{P_{\alpha_{k}}^{k}\right\} \mid \alpha_{k-1} \cdots \alpha_{1}\right) .
$$

A little algebra using (2.26) and (2.27) is enough to show that, for the case of sets of alternatives at a series of times, the step-by-step entropy and the history space entropy are related by

$$
S_{h s}\left(\left\{c_{\alpha}\right\}\right)=S_{s b s}\left(\left\{P_{\alpha_{n}}^{n}\right\}, \cdots,\left\{P_{\alpha_{1}}^{1}\right\}\right)+(N-n) \log _{2} V
$$


That is, they are identical except for a constant factor that is the missing information at the times not specified. Evidently, $S_{s b s}<S_{h s}$ for the coarse-grained sets for which they are both defined.

\section{Dynamically Constrained History Space Entropy}

In constructing of the history space entropy $S_{h s}$, the entropy functional (2.13) is maximized over all probability functions $\widetilde{\mathbf{W}}(\mathbf{x})$ irrespective of whether they conform to the same basic dynamical law. $S_{h s}$ is thus the missing information in histories assuming we are also missing any information about the dynamics.

A dynamical law could be enforced by maximizing $\mathcal{S}(\widetilde{\mathbf{W}})$ only over the $\widetilde{\mathbf{W}}$ that conform to it. For example, by maximizing over the form (2.9), keeping $p_{\eta}(x \mid y)$ fixed, we enforce a particular Markovian dynamical law. The resulting entropy $S_{d c}\left(\left\{c_{\alpha}\right\}\right)$ we call the dynamically constrained history space entropy. The maximum in (2.14) is carried out only over the initial distribution $\tilde{\rho}(x)$ with $\widetilde{\mathbf{W}}(x)$ determined by enforcing the subsequent dynamics explicitly. Evidently, since this is a constrained maximum,

$$
S_{d c}\left(\left\{c_{\alpha}\right\}\right) \leq S_{h s}\left(\left\{c_{\alpha}\right\}\right)
$$

The entropy $S_{d c}\left(\left\{c_{\alpha}\right\}\right)$ is connected to another entropy of histories obtained by applying the Jaynes method used in (1.3) to the initial $\tilde{\rho}(x)$, but constraining the maximum not simply by the requirement that probabilities at one time are reproduced, but probabilities of a whole set of histories. We call this the initial condition entropy $S_{i c}\left(\left\{c_{\alpha}\right\}\right)$.

We can illustrate the construction of $S_{i c}$ in the case of Markovian evolution and a set of histories that is a sequence of sets of alternatives $\left\{P_{\alpha_{k}}^{k}\right\}$ at a series of times $t_{k}, k=1, \cdots, n$. The probabilities of these histories are given by (2.12) which we may conveniently write as

$$
p_{\alpha_{n} \cdots \alpha_{1}}=\operatorname{Tr}\left(C_{\alpha_{n} \cdots \alpha_{1}} \rho\right)
$$


that is, the sum or integral of $\rho\left(x_{0}\right)$ with the functions $C_{\alpha_{n}} \cdots \alpha_{1}\left(x_{0}\right)$ defined by (2.12). We can now carry out the Jaynes construction

$$
\left.S_{i c}\left(\left\{c_{\alpha}\right\}\right)=\left.\max _{\tilde{\rho}} \mathcal{S}(\tilde{\rho})\right|_{\operatorname{Tr}\left[C_{\alpha_{n}}\right.} \ldots \alpha_{1} \tilde{\rho}\right]=\operatorname{Tr}\left[\begin{array}{llll}
C_{\alpha_{n}} & \ldots & \alpha_{1} \rho
\end{array}\right]
$$

for all the histories. The density function which realizes this maximum has the form

$$
\tilde{\rho}(x)=\exp \left[-\sum_{\alpha_{n} \cdots \alpha_{1}} \lambda^{\alpha_{n} \cdots \alpha_{1}} C_{\alpha_{n} \cdots \alpha_{1}}(x)\right]
$$

where the Lagrange multipliers $\lambda^{\alpha_{n} \cdots \alpha_{1}}$ are determined by the conditions

$$
\operatorname{Tr}\left(C_{\alpha_{n}} \cdots \alpha_{1} \tilde{\rho}\right)=\operatorname{Tr}\left(C_{\alpha_{n} \cdots \alpha_{1}} \rho\right) .
$$

The $C_{\alpha_{n} \ldots \alpha_{1}}(x)$ are not projections, and there seems no easy way to evaluate (2.32) and (2.33) explicitly in general. However, $S_{d c}$ and $S_{h s}$ supply upper bounds on $S_{i c}$ as we shall now show.

Write out the entropy functional (2.13) for $\widetilde{\mathbf{W}}$ of the form (2.9) to find after a little algebra

$$
\mathcal{S}(\tilde{\rho})=\mathcal{S}(\widetilde{\mathbf{W}})-\int d x_{0} s\left(x_{0}\right) \tilde{\rho}\left(x_{0}\right)
$$

where $\mathcal{S}(\widetilde{\mathbf{W}})$ is (2.13) for $\mathbf{W}$ of the form (2.9), and

$$
\mathcal{S}(\tilde{\rho})=-\operatorname{Tr}\left(\tilde{\rho} \log _{2} \tilde{\rho}\right)
$$

The entropy $s\left(x_{0}\right)$ is defined by

$$
s\left(x_{0}\right)=-\int d x_{n} \cdots d x_{1} p\left(x_{n} \cdots x_{0}\right) \log _{2} p\left(x_{n} \cdots x_{0}\right),
$$

where

$$
p\left(x_{n}, \cdots, x_{0}\right)=p\left(x_{n} t_{n} \mid x_{n-1} t_{n-1}\right) \cdots p\left(x_{1} t_{1} \mid x_{0} t_{0}\right) .
$$

The function $s\left(x_{0}\right)$ is always positive. Thus

$$
\mathcal{S}(\tilde{\rho}) \leq \mathcal{S}(\widetilde{\mathbf{W}})
$$


for $\tilde{\mathbf{W}}$ of the form (2.9). [Note that the Markovian form of the dynamics in (2.37) is not important; any probability function $p\left(x_{n}, \ldots, x_{0}\right)$ satisfies this inequality.] On maximization over $\tilde{\rho}$ we have the inequalities

$$
S_{i c} \leq S_{d c} \leq S_{h s} .
$$

In particular, if on fine-graining $S_{h s}$ is driven to a low value, then $S_{d c}$ and $S_{i c}$ will be as well. We shall use this in what follows.

\section{BEHAVIOR OF HISTORY SPACE ENTROPY UNDER FINE-GRAINING}

Entropies decrease under fine-graining and increase under coarse-graining. That immediately follows from the Jaynes construction as the discussion leading to (2.16) shows. Usually this well-known behavior is considered for variations in levels of coarsegraining at a given moment of time. However, histories can also be fine-grained in time. For example, if a set of histories is specified by one set of alternatives at a series of times, and another set of histories by the same alternatives at more times, then the second set is a fine-graining of the first.

In this section we examine explicitly the behavior of history space entropy under fine-graining in three one-dimensional models with simple stochastic evolutionary laws. They are: a discrete random walk, continuous diffusion, and Brownian motion. The random walk is the simplest model; diffusion illustrates the modifications necessary for the continuum; and Brownian motion is a simple example of a non-Markovian process. In all cases we consider a finest-grained net of $N$ equally-spaced times so that fine-grained histories are specified by $N$ positions $\left(x_{1}, \cdots, x_{N}\right)$. We consider coarse-grainings in which these positions are grouped into equal intervals of size $\Delta x$ at a series of times spaced by equal intervals $\Delta t$. We then study history space entropy for these coarse-grainings as a function of $\Delta x$ and $\Delta t$. 


\section{A. Random Walk}

We take an initial condition where all histories begin at the initial point $x_{0}=0$

and assume that at each timestep the particle has an equal chance of moving right $(x \rightarrow x+1)$ or left $(x \rightarrow x-1)$ on a discrete spatial lattice. There are then $2^{N}$ finegrained histories with equal probability $1 / 2^{N}$ and all other histories have probability 0 . We assume that the lattice has a large finite size $V$ with periodic boundary conditions relating its ends. The history space entropy is given by (2.17) where $\operatorname{Tr}\left(\mathbf{P}_{\alpha}\right)$ is the number of fine-grained histories in a coarse-grained history $c_{\alpha}$. For all histories with the coarse-graining described above this is:

$$
\operatorname{Tr}\left(\mathbf{P}_{\alpha}\right)=(\Delta x)^{N / \Delta t} V^{N(1-1 / \Delta t)}
$$

Simple as this is, it is clear that as the number of fine-grained histories increases rapidly with the number of times $n=N / \Delta t$, and calculating entropies by summing over all the fine-grained histories in each coarse-grained history rapidly becomes impractical. Instead, we use a Monte Carlo approach: we generate a large sample of fine-grained histories, bin them together into coarse-grained classes, and calculate the entropies from the resulting probability estimates. This technique works in the continuous case as well.

In Figure 1 we plot the history space entropy $S_{h s}$ of the random walk model as a function of the $\Delta x$ and $\Delta t$. We clearly see that the entropy rises steeply when the coarse-graining is increased by increasing $\Delta t$ and more moderately as $\Delta x$ is increased. Increasing $\Delta x$ to $V$ at a fixed time gives the maximal coarse-graining where the only alternatives are $(I, 0)$. Therefore, increasing $\Delta x$ to $V$ for any fixed value of $\Delta t$ will give the maximum possible entropy, associated with the alternative $\mathbf{P}=\mathbf{I}$. That is, from (3.1) (the $-\sum p \log _{2} p$ term vanishes),

$$
S_{h s}^{\max }=N \log _{2} V .
$$




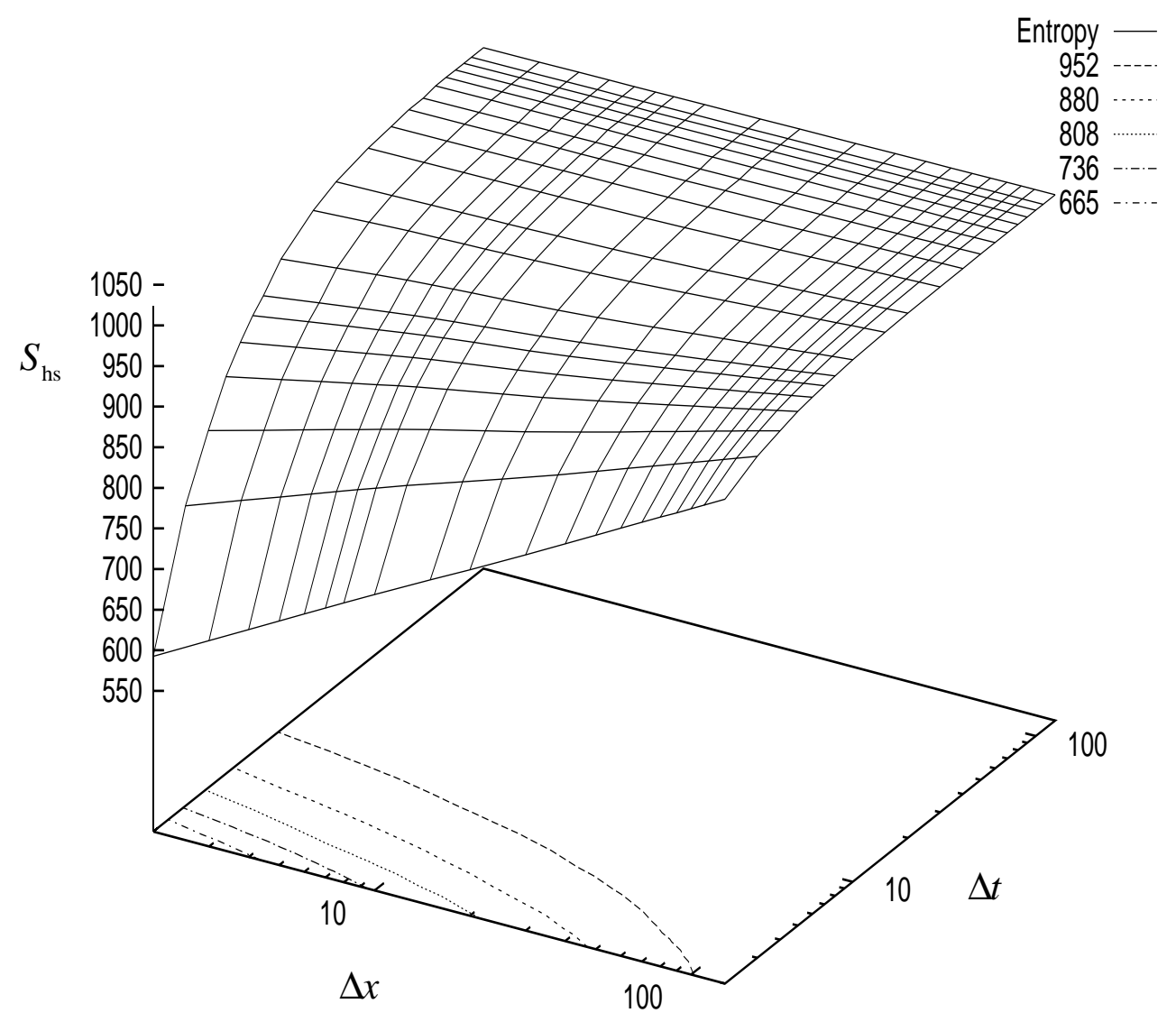

Figure 1: History space entropy, $S_{h s}$, for the discrete random walk as a function of coarse-graining scales $\Delta x$ and $\Delta t$. In this system, a particle begins at $x=0$ on a $1 D$ lattice of 256 points and moves left or right by 1 position with equal probability at each of $N=128$ times. The entropy is measured in bits of missing information. These results were produced by a Monte Carlo simulation with 100,000 random trajectories; because of the rapid rise in $S_{h s}$ with coarse-graining, the $\Delta x$ and $\Delta t$ axes are plotted on a logarithmic scale. 
Increasing $\Delta t$ for fixed $\Delta x$ gives a closely related limit. When $\Delta t$ is at its maximum value of $N, S_{h s}$ is the single time entropy plus $(N-1) \log _{2} V[c f$. (2.28)]. The single time entropy ranges from $N \log _{2} 2$ for $\Delta x=1$ to $N \log _{2} V$ for $\Delta x=V$. Thus, for large $N$ we expect $S_{h s}$ to be essentially $S_{h s}^{\max }$ and that behavior is also illustrated in Figure 1.

The maximum value of $S_{h s}$ for the particular model simulated is $N \log _{2} V$, which in this case is 1024 bits. This is reflected on the plot. At the other extreme, the minimum entropy occurs for $\Delta t=\Delta x=1$, and is $S_{h s}=128$ bits. The finest graining included in Figure 1 is $\Delta t=\Delta x=2$, and we see that $S_{h s}$ has already risen steeply at that point.

\section{B. Continuous Diffusion}

A Markovian diffusion process illustrates the case when $M$ is a continuous space. Take the transition probability to be

$$
p\left(x_{2}, t_{2} \mid x_{1}, t_{1}\right)=\frac{1}{\sqrt{\pi D \Delta t}} \exp \left[-\left(x_{2}-x_{1}\right)^{2} / D \Delta t\right]
$$

where $D$ is a diffusion constant and $\Delta t=t_{2}-t_{1}$. Assume a finite range size $V$, divided into cells of size $\Delta x$, and a total duration for the histories of $t_{f}=N \Delta t$.

Choose $V \gg \sqrt{D t_{f}}$, so that we needn't worry about boundaries. We again assume an initial condition where the particle is initially at $x_{0}$.

Label the intervals of the spatial coarse-graining by an integer $i$, a point lying in the $i^{\text {th }}$ cell if $i \Delta x \leq x<(i+1) \Delta x$. The probability of a particle initially at $x_{0}$ passing through a sequence of $n$ cells $i_{1}, \ldots, i_{n}$ at times $t_{j}=j \Delta t$ is

$$
\begin{aligned}
p\left(i_{1}, \ldots, i_{n}\right) & =\int_{i_{1} \Delta x}^{\left(i_{1}+1\right) \Delta x} d x_{1} \cdots \int_{i_{n} \Delta x}^{\left(i_{n}+1\right) \Delta x} d x_{n} \prod_{j=1}^{n} p\left(x_{j}, j \Delta t \mid x_{j-1},(j-1) \Delta t\right) \\
& =\frac{1}{(\pi D \Delta t)^{n / 2}} \int_{i_{1} \Delta x}^{\left(i_{1}+1\right) \Delta x} d x_{1} \cdots \int_{i_{n} \Delta x}^{\left(i_{n}+1\right) \Delta x} d x_{n} \exp \left[-\sum_{j=1}^{n} \frac{\left(x_{j}-x_{j-1}\right)^{2}}{D \Delta t}\right] .
\end{aligned}
$$


The history space entropy for the continuous case has exactly the same form as (2.17) the discrete case, but it is convenient to make use of a dimensionally invariant form of the entropy, by subtracting a dimensional factor $\log _{2} V^{N}$. Thus, the $\log _{2} \operatorname{Tr}\left(\mathbf{P}_{\alpha}\right)$ term in (2.17) becomes $\log _{2}\left[\operatorname{Tr}\left(\mathbf{P}_{\alpha}\right) / \operatorname{Tr}(\mathbf{I})\right]$. Rather than being an integer, as the discrete case, it is a continuous measure of the coarse-graining of each history. For the coarse-graining described above, with intervals of size $\Delta x$ and $n=t_{f} / \Delta t$ times, we get

$$
\log _{2}\left[\operatorname{Tr}\left(\mathbf{P}_{\alpha}\right) / \operatorname{Tr}(\mathbf{I})\right]=n \log _{2}(\Delta x / V) .
$$

$\Delta x / V<1$, so $\log _{2}\left[\operatorname{Tr}\left(\mathbf{P}_{\alpha}\right) / \operatorname{Tr}(\mathbf{I})\right]<0$ for all but maximally coarse-grained histories.

There are $(V / \Delta x)^{n}$ coarse-grained histories. The $p \log _{2} p$ part of the entropy in (2.17) is maximized in the case when all the histories have equal probabilities. In this case,

$$
\max \sum_{\alpha}\left(-p_{\alpha} \log _{2} p_{\alpha}\right)=n \log _{2}(V / \Delta x),
$$

Taking account of (3.5) we see that 0 is the maximum of $S_{h s}$ so that it is strictly non-positive. This is different from usual definitions of entropy, which are logarithms of large numbers and hence always positive. However, what is important is the change in $S_{h s}$ under coarse-graining or refinement, not its absolute value.

We can gain some insight by looking at the limiting behavior of $S_{h s}$ for different levels of coarse-graining. Consider first the coarse-grained limit where $\Delta x \rightarrow V$. As $\Delta x$ becomes large compared to $\sqrt{D t_{f}}$, it becomes highly improbable that the particle will ever diffuse outside of a single cell $i$. Thus, in this limit, one history dominates with a probability $p \approx 1$ while the others are suppressed, $p \approx 0$, and the $-\sum p \log _{2} p$ part of the entropy vanishes. At the same time, the term $\log _{2}\left[\operatorname{Tr}\left(\mathbf{P}_{\alpha}\right) / \operatorname{Tr}(\mathbf{I})\right]=$ $n \log _{2}(\Delta x / V)$ approaches 0 as well, so this maximal coarse-graining in $x$ leads to $S_{h s} \rightarrow 0 ; S_{h s}$ is maximized by maximal coarse-graining in $x$.

Let us go now to the opposite limit, where $\Delta x \ll V$. We can now label the interval $i_{j}$ by the value $x_{j}$ centered in that interval. The probability to go from $x_{j-1}$ to $x_{j}$ is 


$$
p\left(x_{j} \mid x_{j-1}\right)=\frac{\Delta x}{\sqrt{\pi D \Delta t}} \exp \left[-\frac{\left(x_{j}-x_{j-1}\right)^{2}}{D \Delta t}\right] .
$$

The $p \log _{2} p$ term for a single history is then

$$
\begin{aligned}
& -p\left(x_{1}, \ldots, x_{n}\right) \log _{2} p\left(x_{1}, \ldots, x_{n}\right)=-\frac{n}{2} \log _{2}\left(\frac{\Delta x^{2}}{\pi D \Delta t}\right) p\left(x_{1}, \ldots, x_{n}\right) \\
& +\left(\log _{2} e\right)\left(\sum_{j} \frac{\left(x_{j}-x_{j-1}\right)^{2}}{D \Delta t}\right)\left(\frac{\Delta x^{2}}{\pi D \Delta t}\right)^{n} \exp \left[-\sum_{j} \frac{\left(x_{j}-x_{j-1}\right)^{2}}{D \Delta t}\right] .
\end{aligned}
$$

Summing over all histories is the same as summing the above expression over all the $x_{j}$. These sums can be approximated by integrals, which are readily evaluated to yield

$$
\sum_{\alpha}\left(-p_{\alpha} \log _{2} p_{\alpha}\right) \approx n \log _{2}\left(\frac{\sqrt{\pi D t_{f}}}{\Delta x}\right)-\frac{n}{2}\left(\log _{2} n-1\right)
$$

Adding the expression for $\log _{2} \operatorname{Tr}\left(\mathbf{P}_{\alpha}\right) / \operatorname{Tr}(\mathbf{I})$ from (3.5) gives for the entropy

$$
S_{h s} \approx n \log _{2}\left(\frac{\sqrt{\pi D t_{f}}}{V}\right)-\frac{n}{2}\left(\log _{2} n-1\right)<0
$$

i.e., $S_{h s}$ approaches a constant negative value in the limit of small $\Delta x$ for a fixed $\Delta t$.

Suppose now that we hold $\Delta x$ fixed and vary the coarse-graining in $t$. If we go to the maximum coarse-graining $\Delta t=t_{f}$, we return to the case of alternatives at a single time. If the probability of the particle being in the interval $i$ is $p_{i}$, the entropy is just

$$
S_{h s}=-\sum_{i} p_{i} \log _{2} p_{i}+\log _{2}(\Delta x / V)
$$

differing from the usual single-time entropy only by a constant. 


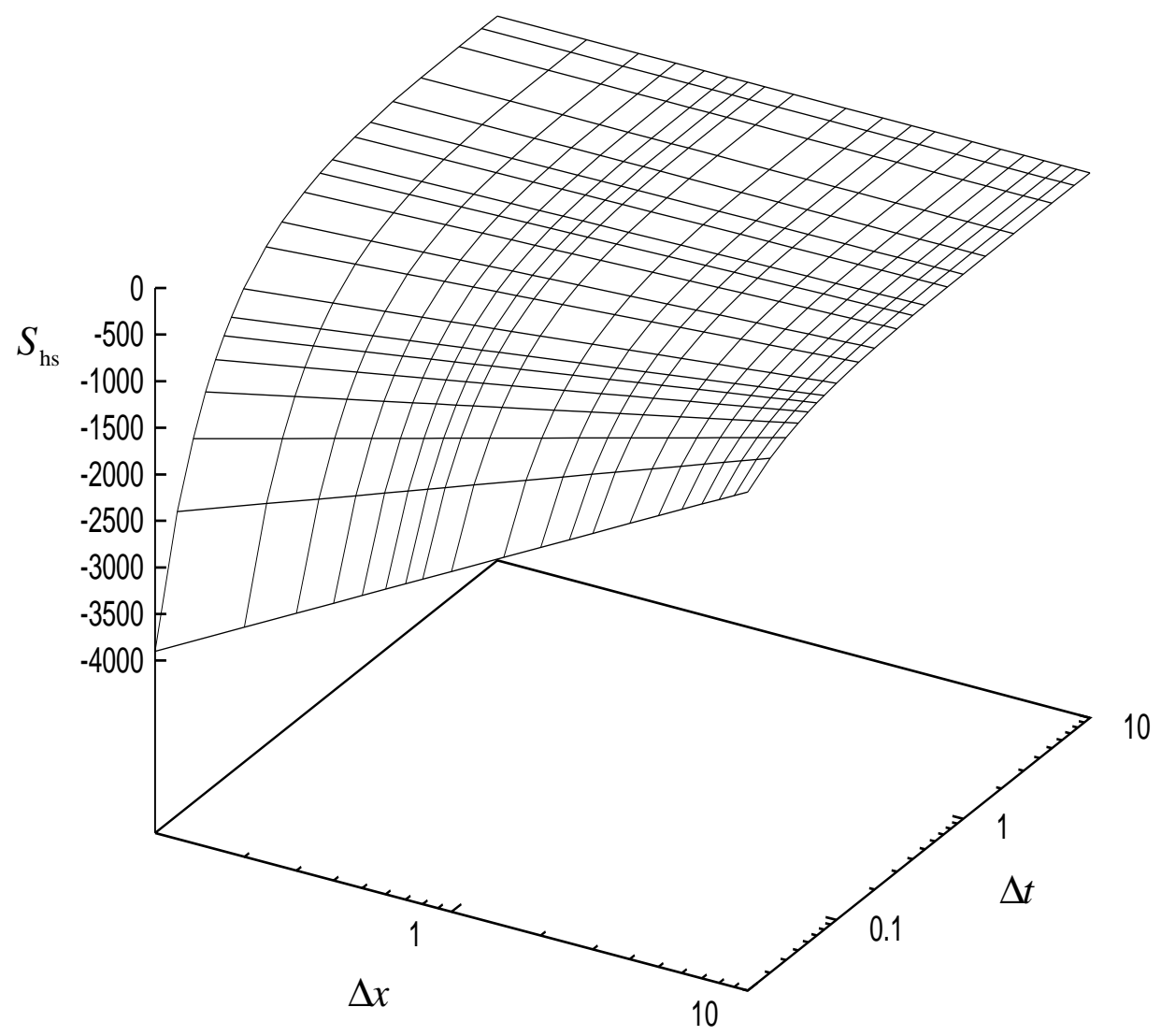

Figure 2: History space entropy $S_{h s}$ for continuous diffusion as a function of coarse-graining scales $\Delta x$ and $\Delta t$, in bits. All particles begin at $x=0$ on a $1 D$ manifold of length $V=20$, and spread with diffusion constant $D=1$ through a finest-grained net of $N=1024$ times with minimal timestep $\eta=0.01$. The finest-grained cell size is $\Delta x=0.1$. We have subtracted off the the maximum entropy $N \log _{2} V$ to render our results invariant under dimensional rescaling and refinements in time; the maximum entropy is thus 0 , and $S_{h s}$ is not bounded below. These results were produced by a Monte Carlo simulation with 10,000 random trajectories. 
If instead we refine the description in time the result is quite different. As the timestep $\Delta t$ becomes small compared to $\Delta x^{2} / D$, the probability of a particle moving from one interval to another in a timestep becomes small as well. Beyond that point, refining the description of the system in time does not increase the actual number of alternative histories with non-zero probabilities. Thus,

$$
-\sum p \log _{2} p \rightarrow \text { const. }
$$

The $\log _{2}\left[\operatorname{Tr}\left(\mathbf{P}_{\alpha}\right) / \operatorname{Tr}(\mathbf{I})\right]=n \log _{2}(\Delta x / V)$ term, however, does change as we increase $n$. Because this term is negative, as we increase the fine-graining in $t$, the history space entropy $S_{h s}$ decreases without limit.

In both $x$ and $t$, the entropy is diminished by making the description more finegrained. Thus, we expect the same behavior as in the simple random walk: the entropy $S_{h s}$ (and thus, all other measures of entropy for histories that we have considered) will be minimized by the most fine-grained description. We performed a numerical calculation to generate the entropy plot in Figure 2. Note that the qualitative behavior is exactly the same as in Figure 1.

\section{Brownian motion}

In the previous examples, we assumed an explicitly Markovian time-evolution. If we relax that assumption and suppose that the probability of a history $p\left(x_{1}, \ldots, x_{n}\right)$ does not have the form $p\left(x_{n} \mid x_{n-1}\right) \cdots p\left(x_{2} \mid x_{1}\right) p\left(x_{1}\right)$ are our conclusions affected?

As a simple example of a non-Markovian process, consider a particle undergoing Brownian motion. In addition to inertia and dissipation, the particle is subjected to a stochastic force. We can write a stochastic differential equation for its motion in Itô form:

$$
\begin{aligned}
& d x=(p / m) d t, \\
& d p=-2 \Gamma p d t+a d \xi,
\end{aligned}
$$


where $d \xi$ is a stochastic differential variable with zero mean and variance $d t$,

$$
\mathrm{M}(d \xi)=0, \quad \mathrm{M}\left(d \xi^{2}\right)=d t
$$

This stochastic equation corresponds to a Fokker-Planck equation for probability densities $\rho(x, p, t)$ in phase space [13]:

$$
\frac{d \rho}{d t}(x, p)=-\left(\frac{p}{m}\right) \frac{\partial \rho}{\partial x}(x, p)+2 \Gamma \frac{\partial}{\partial p} p \rho(x, p)+\frac{a^{2}}{2} \frac{\partial^{2} \rho}{\partial p^{2}}(x, p) .
$$

We can enumerate a set of coarse-grained histories for Brownian motion just as we did for the continuous random walk, dividing up the range $V$ into cells of size $\Delta x$ and dividing the total time of the histories $t_{f}$ into $n$ steps of $\Delta t$ each. An individual coarse-grained history consists of all fine-grained histories which pass through a given set of intervals $i_{1}, \ldots, i_{n}$ at times $t_{j}=j \Delta t$.

Histories of $x(t)$ are not Markovian because of the existence of the inertia term $-(p / m) \partial \rho / \partial x$ in (3.15). However, looked at over relatively long times $\Delta t \gg 1 / \Gamma$ the inertia becomes unimportant, as dissipation dominates. On these long timescales, the system is well approximated by the continuous diffusion model (3.3) with $D=$ $a^{2} / 8 \Gamma^{2} m^{2}$. On very short timescales, by contrast, inertia dominates. The particle drifts at a near-constant velocity, only slightly deflected by dissipation and noise.

We see that the same arguments we used in the case of continuous diffusion apply to this case with little modification. Fine-graining in $t$ reduces the entropy without limit. Fine-graining in $x$ is a little less clear, but a similar argument can be made. In the limit of fine-grained $x$, we can approximate the probability of a history as

$$
p\left(x_{1}, \ldots, x_{n}\right)=\left(\Delta x / Q_{0}\right)^{n} f\left(x_{1}, \ldots, x_{n}\right)
$$

where $f\left(x_{1}, \ldots, x_{n}\right)$ is dimensionless, and $Q_{0}$ is a constant with units of length which depends on $\Delta t$ but not $\Delta x$. We can replace the sum over all histories in (2.17) with $n$ integrals over the $x_{j}$, and get 


$$
\begin{aligned}
S_{h s}= & -\frac{1}{Q_{0}^{n}} \int d x_{1} \cdots d x_{n} f\left(x_{1}, \ldots, x_{n}\right) \log _{2} f\left(x_{1}, \ldots, x_{n}\right) \\
& -n \log _{2}\left(\Delta x / Q_{0}\right)+n \log _{2}(\Delta x / V) \\
\equiv & S_{0}+n \log _{2}\left(Q_{0} / V\right)
\end{aligned}
$$

where $S_{0}$ has no $\Delta x$ dependence. Since the $\Delta x$ dependence has dropped out completely, we see that in this case as well the entropy approaches a constant as we fine-grain in $x$.

In Figure 3 we show the numerical results for the entropy of coarse-grained histories of the Brownian motion model as a function of coarse-graining in $x$ and $t$. This graph clearly shows essentially the same behavior of $S_{h s}$ with coarse-graining as in Figures 1 and 2 . 


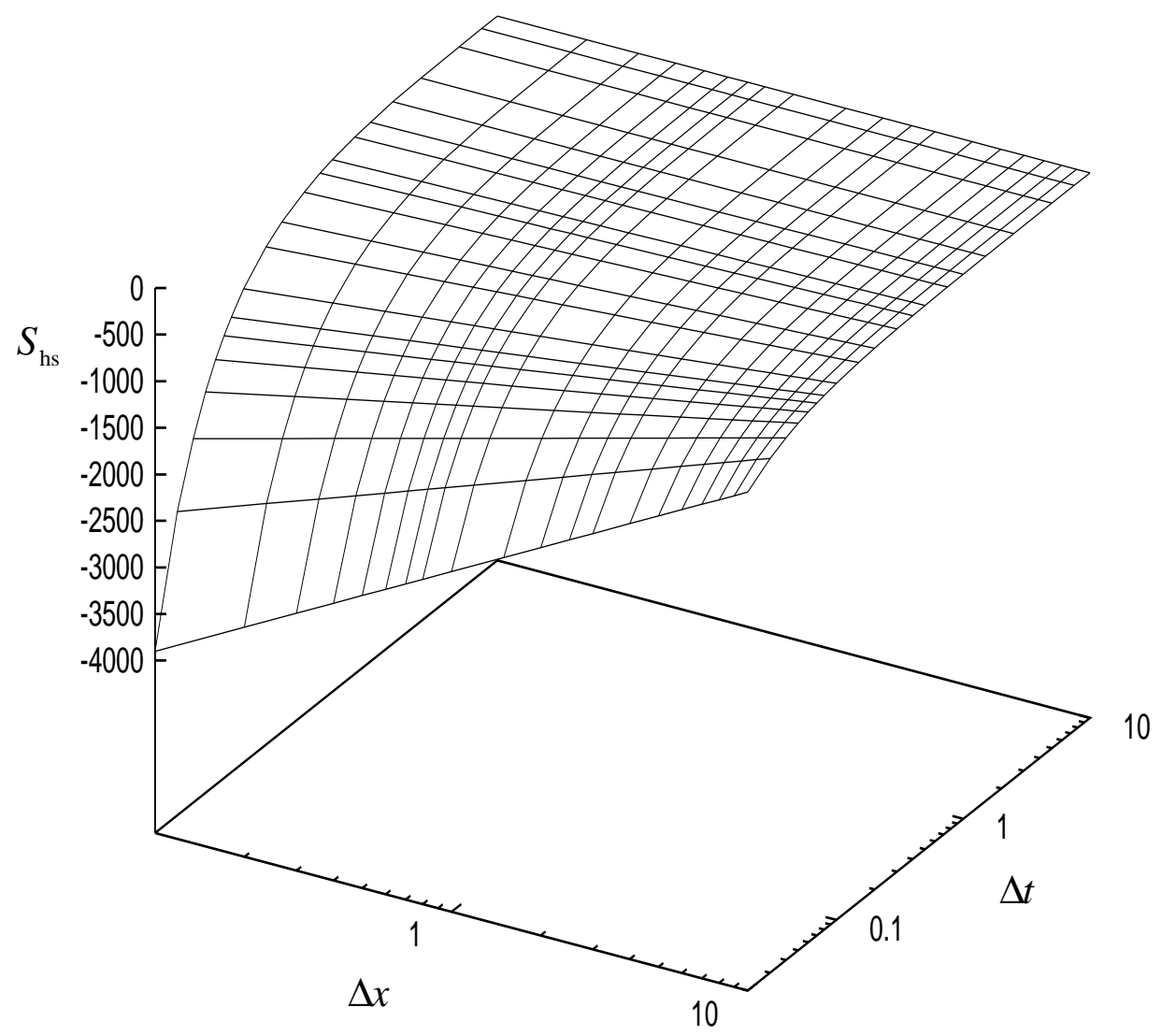

Figure 3: History space entropy, $S_{h s}$, for Brownian motion as a function of coarse-graining scales $\Delta x$ and $\Delta t$, in bits. All particles begin with $(x, p)=(0,0)$ on a $1 D$ manifold of length $V=20$, with dissipation $2 \Gamma=1$, noise strength $a=1$, and mass $m=1$. The finest-grained net of times and minimum cell size are as in Figure 2, and the same conventions are used here in displaying $S_{h s}$. These results were produced by a Monte Carlo simulation with 10,000 random trajectories. 


\section{THE SECOND LAW FOR HISTORIES}

\section{A. The Increase of Entropies}

The familiar second law of thermodynamics concerns the behavior of the entropy of a fixed set of coarse-grained alternatives at a moment of time as this time is varied. We shall call such entropies "single-time entropies".

If the value of a single-time entropy at some particular time $t_{0}$ is all that is known about a system, and if that value is much lower than the maximum (equilibrium) value, then that entropy will subsequently tend to increase for most dynamical laws of interest. If the dynamical law is time symmetric about $t_{0}$, then the approach to equilibrium will also be symmetric about $t_{0}$. However, it is not just this statistical tendency to approach equilibrium that is usually meant by the second law of thermodynamics. Rather, it is the general increase in entropy of suitable coarse-grained descriptions of the universe since the big bang. In particular, what is meant is that, for the most part, certain entropies of presently isolated systems are increasing in the same direction of time. The time-asymmetric increase of these entropies of the universe arises from a cosmological initial condition at which those entropies were low. As Boltzmann put it "The second law of thermodynamics can be proved from

the mechanical theory if one assumes that the present state of the universe ... started to evolve from an improbable state" [14..

The entropies that are most useful in chemistry and physics are associated with quasiclassical coarse-grainings which fix the values of averages over suitable volumes of densities of approximately conserved quantities such as energy, momentum, and abundances of chemical and nuclear species. Their utility arises from the approximate conservation. The small volumes over which the averages are taken reach local equilibrium on short time scales, leaving the approach to equilibrium between volumes to be described by phenomenological equations such as the Navier-Stokes equation over longer time scales. The single-time entropy of these coarse-grainings is low in 
the early universe leading to a general tendency to increase.

Statements of the second law often refer to the increase of "the" entropy as though there were only one possible coarse-grained description for which it holds. What is meant by "the" entropy is usually the single-time entropy of the alternatives defining the quasiclassical realm of everyday experience described above. However, we should expect the general increase of the entropy of any set of coarse-grained alternatives which is low in the initial moments of the universe. To give just one example, the single-time entropy of a set of quasiclassical alternatives $\left\{P_{\alpha}\right\}$ increases with time when conditioned on various other quasiclassical alternatives $\left\{P_{\beta}\right\}$. Indeed, such entropies

$$
S\left(\left\{P_{\alpha}\right\}, t \mid \beta, t^{\prime}\right)
$$

are the ones of practical interest. The entropy of a gas inside a piston is the entropy of alternatives referring to the gas given the configuration of the piston. There are thus a variety of coarse-grainings and conditions for which the missing information increases with time.

\section{B. The Increase in History Entropies}

Sets of alternative, coarse-grained histories provide more general coarse-grained descriptions of the universe than sets of coarse-grained alternatives at merely one time. The corresponding entropies of histories should also increase with time if they are low at the time of the system's initial condition. For example, consider a set of

histories consisting of a series of alternatives $\left\{P_{\alpha_{n}}^{n}\right\}, \cdots,\left\{P_{\alpha_{1}}^{1}\right\}$ at a sequence of time $t_{1}, \cdots, t_{n}$ giving a histories entropy

$$
S_{h s}\left(\left\{P_{\alpha_{n}}^{n}\right\}, t_{n}, \cdots,\left\{P_{\alpha_{1}}^{1}\right\}, t_{1}\right)
$$

If $S_{h s}$ is initially low, and these times are all translated forward by an amount $T$, we would expect 


$$
S_{h s}\left(\left\{P_{\alpha_{n}}^{n}\right\}, t_{n}+T ; \cdots ;\left\{P_{\alpha_{1}}^{1}\right\}, t_{1}+T\right)
$$

to increase with $T$.

A proof of the second law even for single entropies exists only for highly idealized situations. [ That is partly because entropy does not monotonically increase but fluctuates about an increasing trend. We can therefore hardly expect a mathematical proof of the increase of (4.3) with $T$. However, the connection of $S_{h s}$ with the stepby-step entropy supports this in the following way.

Consider histories consisting of alternatives at just two times $t_{1}$ and $t_{2}$. Then from (2.27) and (2.28)

$$
\begin{aligned}
S_{h s}\left(\left\{P_{\alpha_{2}}^{2}\right\}, t_{2} ;\left\{P_{\alpha_{1}}^{1}\right\}, t_{1}\right) & =\sum_{\alpha_{1}} p\left(\alpha_{1}\right) S\left(\left\{P_{\alpha_{2}}^{2}\right\}, t_{2} \mid \alpha_{1}, t_{1}\right) \\
& +S\left(\left\{P_{\alpha_{1}}^{1}\right\}, t_{1}\right)+\text { const . }
\end{aligned}
$$

where the constant is independent of $t_{1}, t_{2}$ and the alternatives. As $t_{1}$ increases, the second term in (4.4) increases. That is just the usual second law. The first term can also be expected to increase as both $t_{1}$ and $t_{2}$ move away from a low entropy initial condition, provided $P_{\alpha_{1}}^{1}$ is sufficiently coarse-grained that the initial condition plays an important role in determining future probabilities.

The sequence of times necessary to specify a set of histories presents a variety of possibilities for investigating the change in entropy. We have already discussed a uniform translation of all the times. However, we could also discuss increasing the separation between the times. For example, in the two time case of (4.4), $S_{h s}$ increases as $t_{1}$ is fixed and $t_{2}-t_{1}$ increases. Indeed, that is just a special case of the usual second law $[c f .(4.1)]$.

\footnotetext{
${ }^{2}$ See, e.g. 15
} 


\section{The Urn Model}

An exactly soluble model which nicely illustrates the increase in history space entropy is the urn model of P. and T. Ehrenfest [16]. The model concerns $2 R$ numbered balls, each of which is in one of two urns, $A$ or $B$. The system evolves through $N$ discrete time steps. At each time a number from 1 to $2 R$ is chosen and that ball is moved from its present urn to the other. Fine-grained histories are specified by giving the urn containing each ball at each of the $N$ times. A simple kind of coarse-grained history specifies the number of balls in one urn, say $A$, at one time $t$. The kind of multi-time, coarse-grained histories we shall study are specified by giving the number of balls in $A,\left(n_{1}, \cdots, n_{n}\right)$ at a sequence of the $N$ times $t_{1}, \cdots, t_{n}$.

The probabilities relevant for constructing the entropies can be worked out [16,17. The probability of a transition from one time to the next is:

$$
p\left(n_{j+1}, t_{j+1} \mid n_{j}, t_{j}\right)=\frac{2 R-n_{j}}{2 R} \delta_{n_{j+1}, n_{j}+1}+\frac{n_{j}}{2 R} \delta_{n_{j+1}, n_{j}-1} .
$$

Given that the number of balls in urn $A$ is $n_{0}$ at time $t_{0}$, the probability that $A$ will contain $n_{j}$ balls at time $t_{j}$ is:

$$
p\left(n_{j}, t_{j} \mid n_{0}, t_{0}\right)=(-1)^{j} 2^{-2 R} \sum_{l=-R}^{R}(l / R)^{j} C_{n_{j}}^{l} C_{R+l}^{R-n_{0}},
$$

where the coefficients $C_{k}^{l}$ are defined by the identity

$$
(1-z)^{R-l}(1+z)^{R+l} \equiv \sum_{k=0}^{2 R} C_{k}^{l} z^{k} .
$$

All the rest of the probabilities we shall need are easily constructed from (4.5) and (4.6).

Consider, by way of example, the history space entropy for the set of histories specified by giving the number of balls in $A$ at two times $t_{j}$ and $t_{j+m}$ assuming an initial condition in which $n_{0}$ balls are in $A$ at $t_{0}$. We call these "two time histories" for short. From (2.17) this is 


$$
\begin{aligned}
S_{h s}\left(\left\{n_{j+m}, n_{j}\right\}\right) & =-\sum_{n_{j+m}, n_{j}} p\left(n_{j+m}, n_{j} \mid n_{0}\right) \log _{2} p\left(n_{j+m}, n_{j} \mid n_{0}\right) \\
& +\sum_{n_{j+m}, n_{j}} p\left(n_{j+m}, n_{j} \mid n_{0}\right) \log _{2}\left(\begin{array}{c}
2 R \\
n_{j+m}
\end{array}\right)\left(\begin{array}{c}
2 R \\
n_{j}
\end{array}\right) \\
& +(N-2) \log _{2}\left(2^{2 R}\right) .
\end{aligned}
$$

The probability $p\left(n_{j+m}, n_{j} \mid n_{0}\right)$ is obtained by multiplying (4.6) by a factor of (4.8) for each of the $m$ times between $t_{j}$ and $t_{j+m}$ and summing over the intermediate values of $n_{k}, j<k<m$. There are $2^{2 R}$ ways of arranging the balls among the urns at each time so that, and a binomial coefficient gives the number of arrangements of balls in which $n$ are in urn $A$. Thus,

$$
\operatorname{Tr}(I)=2^{2 R} \quad, \quad \operatorname{Tr}\left(P_{n}\right)=\left(\begin{array}{c}
2 R \\
n
\end{array}\right) .
$$

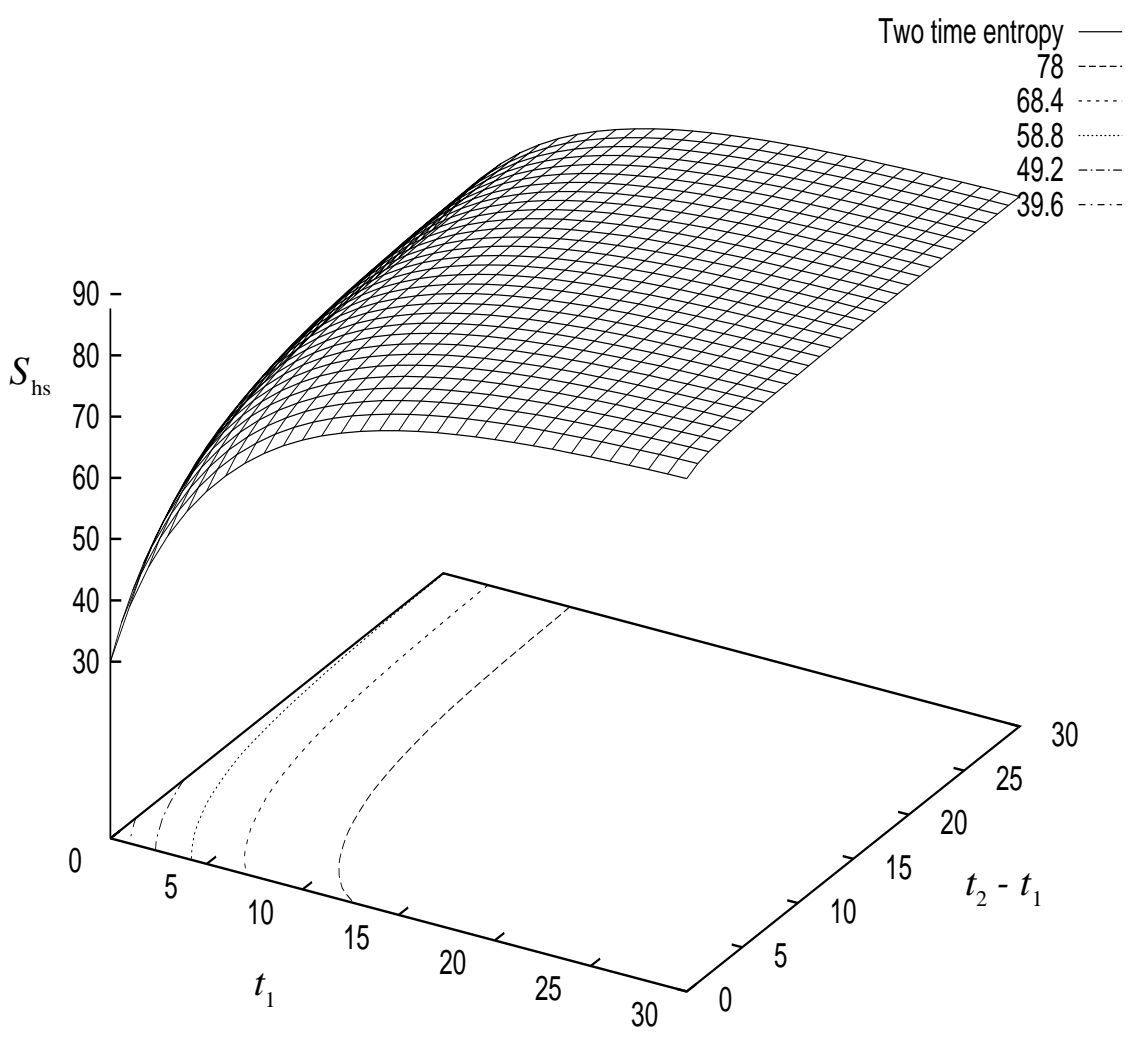


Figure 4: History space entropy, $S_{h s}$, for two time histories of the Ehrenfest urn model as a function of $t_{1}$ and $m=t_{2}-t_{1}$, in bits. In the case shown there are $2 R=30$ numbered balls distributed between urns $A$ and $B$, with all the balls initially in urn $A$. In Figures 4 and 5 we have set the total number of fine-grained times arbitrarily at $N=3$; a larger, more realistic number would merely add a constant displacement to $S_{h s}$.

It takes of order $2 R$ time steps to share information among the $2 R$ balls, and that is the order of characteristic relaxation time for entropies to increase to their maximum value [17]. This is the case for the entropies of two time histories as $t_{1}$ and $t_{2}$ are increased keeping their difference constant; this was suggested by (4.4) and shown by Figure 4. The relation (4.4) shows that the maximum value (not including the neglected times) is roughly twice the maximum entropy for single time coarse-grainings of this type.

This relation also indicates that $S_{h s}$ should grow with the same characteristic relaxation time as $t_{2}-t_{1}$ is increased, keeping $t_{1}$ fixed. The increase comes from the first term in (4.4). Again, the maximum value reached lies between one and two times the maximum for single-time coarse-grainings by the number of balls in one urn. This behavior is also evident in Figure 4 (though for large $t_{1}$ the increase is almost saturated at the initial time).

Increasing the number of times included in each history is a fine-graining. At a given value of $t_{1}$, the entropy should decrease as more times are included. This behavior is illustrated in Figure 5 for $2 R=n_{0}=30$. This shows the behavior of one, two, and three time history space entropies as a function of $t_{1}$ where $t_{2}=t_{1}+1$, and $t_{3}=t_{1}+2$. All the entropies increase to maximum values on roughly the time scale $R$. Asymptotically from (4.4), the entropies behave like

$$
S_{1}\left(t_{1}\right)-c
$$

where $S_{1}$ is the single-time entropy and $c$ is independent of $t_{1}$ for the urn model but 
depends on the number of times and the values of the time differences.

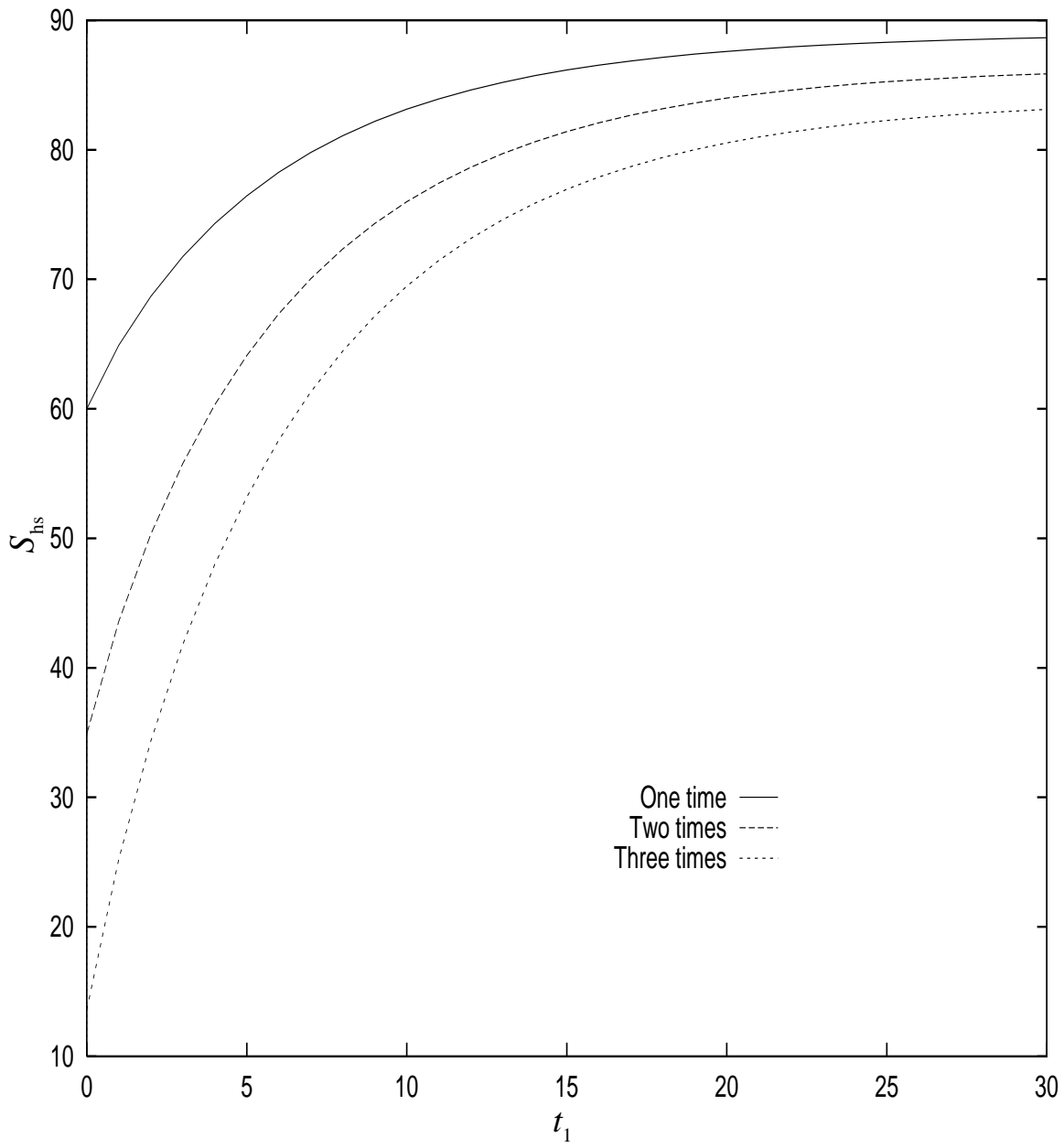

Figure 5: History space entropy, $S_{h s}$, for one, two, and three time histories of the Ehrenfest urn model versus the first specified time $t_{1}$, in bits. The times of the two and three time histories are separated by single timesteps. The parameters and initial conditions are the same as in Figure 4.

\section{QUANTUM HISTORY SPACE ENTROPY}

Isham and Linden posited their family of entropies (2.23) on the basis of the property that they decrease under fine-graining. We were able to show that the 
classical analogs could be derived from a Jaynes construction for the case $x=1$. In this section we show that quantum history space entropy can be similarly derived as a preliminary to a more general discussion of its connection with other entropies.[

Consider a set of decoherent alternative histories $\left\{c_{\alpha}\right\}$, each history with a probability $p_{\alpha}$ and represented in history space by a projector $\mathbf{P}_{\alpha}$. Define an entropy functional on history space operators $\widetilde{\mathbf{W}}$ by

$$
\mathcal{S}(\widetilde{\mathbf{W}})=-\operatorname{Tr}\left(\widetilde{\mathbf{W}} \log _{2} \widetilde{\mathbf{W}}\right)
$$

Then maximize $\mathcal{S}(\widetilde{\mathbf{W}})$ over all $\widetilde{\mathbf{W}}$ for which 5.1 is real, subject to the condition that

$$
\operatorname{Tr}\left(\mathbf{P}_{\alpha} \widetilde{\mathbf{W}}\right)=p_{\alpha}
$$

The result is that the maximum is given by

$$
\widetilde{\mathbf{W}}=\sum_{\alpha} p_{\alpha} \frac{\mathbf{P}_{\alpha}}{\operatorname{Tr}\left[\mathbf{P}_{\alpha}\right]},
$$

and the entropy is:

$$
S_{h s}\left(\left\{c_{\alpha}\right\}\right)=-\sum_{\alpha} p_{\alpha} \log _{2} p_{\alpha}+\sum_{\alpha} p_{\alpha} \log _{2} \operatorname{Tr}\left(\mathbf{P}_{\alpha}\right) .
$$

analogous to (2.17).

The Jaynes construction immediately makes clear why the $x=1$ history space entropy decrease on fine-graining. There are more conditions constraining the maximum in (2.14) in a fine-graining of a set than in the set itself. The maximum can therefore only be lower. For other values of $x$ it is sufficient to note that

$$
I_{x}\left(\left\{c_{\alpha}\right\}\right)=S_{h s}\left(\left\{c_{\alpha}\right\}\right)-\operatorname{Tr}(\mathbf{I})+(x-1) \sum_{\alpha} p_{\alpha} \log _{2}\left[\operatorname{Tr}\left(\mathbf{P}_{\alpha}\right)\right]
$$

This too decreases with fine-graining, as follows from the result for $I_{1}$ and the convexity of the logarithm.

\footnotetext{
${ }^{3}$ The authors have benefited from many discussions with M. Gell-Mann on this issue.
} 
Thus, history space entropy can be given a unified construction through a Jaynes procedure both classically and quantum mechanically. What can be done classically but not quantum mechanically is to express the probabilities for all decoherent histories in the form

$$
p_{\alpha}=\operatorname{Tr}\left(\mathbf{P}_{\alpha} \mathbf{W}\right)
$$

for one positive operator $\mathbf{W}$, independent of the set of alternatives. There is no quantum mechanical analog of (2.9). Were there one, quantum mechanics would be equivalent to a classical stochastic theory. It is possible to find history space operators W which reproduce the probabilities $p_{\alpha}$ through (5.6) for any decoherent set. For example, valid expressions for the probabilities of decoherent histories like

$$
p_{\alpha}=\operatorname{Tr}\left(P_{\alpha_{n}}^{n}\left(t_{n}\right) \cdots P_{\alpha_{1}}^{1}\left(t_{1}\right) \rho\right)
$$

can be transcribed into history space using the identity [12]

$$
\operatorname{Tr}_{\mathcal{H}}\left(A_{1} \cdots A_{n}\right)=\operatorname{Tr}_{\otimes^{k \mathcal{H}}}\left[\left(A_{1} \otimes \cdots \otimes A_{n}\right) S\right]
$$

where

$$
S\left|v_{1}\right\rangle \otimes \cdots \otimes\left|v_{k}\right\rangle=\left|v_{k}\right\rangle \otimes\left|v_{1}\right\rangle \otimes \cdots \otimes\left|v_{k-1}\right\rangle
$$

However, the resulting W's are not positive, even when they can be arranged to be Hermitean. For this reason, even though quantum analogs of $S_{d c}\left(\left\{c_{\alpha}\right\}\right)$ and $S_{i c}\left(\left\{c_{\alpha}\right\}\right)$ can be defined, the derivations of the inequalities relating them to $S_{h s}\left(\left\{c_{\alpha}\right\}\right)$ like (2.39) do not immediately generalize to quantum mechanics.

\section{CONCLUSIONS}

Information is contained not only in sets of alternatives at a single moment of time, but more generally in sets of alternative histories - sequences of sets of alternatives at a series of times. A variety of measures of the information in histories are available. In 
this paper we have provided a unified construction of all of these through the Jaynes procedure. It follows from these constructions that these entropies decrease under fine-graining and increase under coarse-graining. We illustrated this in a few simple models.

We expect entropies for histories to share other common properties analogous to the usual second law of thermodynamics. In particular, the entropy of a set of histories should increase as that set is translated forward in time away from a low entropy initial condition. We illustrated this with the classical urn model, but expect it to hold for more realistic dynamical laws, both classically and quantum mechanically.

General sets of alternative coarse-grained histories will not exhibit deterministic correlations in time in a classical stochastic theory. However, sufficiently coarsegrained sets of histories may exhibit deterministic behavior. For example, the unpredictable motion of single atoms yields nearly deterministic laws for the hydrodynamic variables of pressure, temperature, and density. Characterizing the level of determinism is an interesting question related to the search for measures of classicality in quantum theory. It is clear from our discussion that no entropy of histories is a measure of determinism. Entropy is reduced by fine-graining, and the finest-grained histories are not deterministic. In quantum theory we can, therefore, not expect an entropy of of histories, by itself, to be a measure of classicality.

\section{ACKNOWLEDGMENTS}

We would like to thank Carl Caves and Murray Gell-Mann for useful discussions. The work of T. Brun was supported in part by NSF grant PHY94-07194 and that of J.B. Hartle by NSF grants PHY95-07065 and PHY94-07194. 


\section{REFERENCES}

[1] For a classic introduction to statistical mechanics from an information theoretic point of view see A. Katz, Principles of Statistical Mechanics: The Information Theory Approach, W.H. Freeman, San Francisco, (1967) and D.N. Zubarev, Nonequilibrium Statistical Thermodynamics, ed. by P. Gray and P.J. Shepherd, Consultants Bureau, New York, (1974).

[2] See E.T. Jaynes, Papers on Probability, Statistics, and Statistical Mechanics, ed. by R.D. Rosenkrantz, D. Reidel, Dordrecht (1983).

[3] S. Lloyd and H. Pagels, Annals of Physics, 188, 186 (1988).

[4] M. Gell-Mann and J.B. Hartle, in Complexity, Entropy, and the Physics of Information, SFI Studies in the Sciences of Complexity, Vol. VIII, ed. W. Zurek, Addison Wesley, Reading, MA (1990) or in Proceedings of the 3rd International Symposium on the Foundations of Quantum Mechanics in the Light of New Technology ed. by S. Kobayashi, H. Ezawa, Y. Murayama, and S. Nomura, Physical Society of Japan, Tokyo (1990).

[5] C.J. Isham and N. Linden, Phys. Rev. A, 55, 4030 (1997); quant-ph/9612035.

[6] M. Gell-Mann and J.B. Hartle, "Strong Decoherence," to be published in the Proceedings of the 4th Drexel Symposium on Quantum Non-Integrability - The Quantum-Classical Correspondence, Drexel University, September 8-11, 1994, ed. by D.-H. Feng and B.-L. Hu, International Press, Boston/Hong-Kong; grqc/9509054.

[7] M. Gell-Mann and S. Lloyd, Complexity, 2, 44 (1996).

[8] J.B. Hartle, "Spacetime Quantum Mechanics and the Quantum Mechanics of Spacetime" in Gravitation and Quantizations, Proceedings of the 1992 Les Houches Summer School, ed. by B. Julia and J. Zinn-Justin, Les Houches Summer School Proceedings Vol. LVII North Holland, Amsterdam, (1995); gr-qc/9304006. [9] P. Gaspard, Chaos, Scattering, and Statistical Mechanics Cambridge University 
Press, Cambridge, UK, 1998).

[10] C. Isham, J. Math. Phys., 23, 2157 (1994).

[11] C.J. Isham and N. Linden, J. Math. Phys., 35, 5452 (1994), gr-qc/9405029.

[12] C.J. Isham, N. Linden, and S. Schreckenberg, J. Math. Phys., 35, 6360 (1994); qr-qc/9406015.

[13] H. Hasegawa and H. Ezawa, Supp. Prog. Theor. Phys., 69, 41 (1980).

[14] L. Boltzmann, Ann. Physik, 60, 392, (1897).

[15] H. Spohn, Large Scale Dynamics of Interacting Particles, Springer Verlag, Berlin (1991).

[16] P. and T. Ehrenfest, Phys. Z., 8, 311 (1907).

[17] M. Kac, Probability and Related Topics in Physical Sciences, Interscience Publishers, Ltd., London (1959). 